\title{
Sudden death due to metastasizing intravenous leiomyoma: a case report
}

\author{
Filippo Milano ${ }^{1}$, Filippo Borri², Elena Bonanno ${ }^{2}$, Stefania Urso ${ }^{1}$, Giuseppe Alessio Messano, \\ Luigi Tonino Marsella ${ }^{2}$
}

Abstract: Object of the present article is the case of a 43 year-old woman that, about half an hour later experiencing a temporary loss of consciousness during a family trip, suddenly died. There was nothing relevant in the anamnestic data apart that she underwent a similar lipothymic attack three days before. The significative autopsy findings were a thrombotic mass occupying the whole lumen of the inferior vena cava, extending for all the vein length to the heart, and a voluminous uterine neoformation, with intramural extension. Histological investigation revealed a metastasizing intravenous leiomyoma, likely to originate from a pre-existent uterine leiomyoma.

The purpose of this article is to describe such a uncommon metastasizing intravenous leiomyoma case recognized through a joint approach of legal medicine investigations and histopatologic examinations.

Key Words: metastasizing intravenous leiomyoma, sudden death, autopsy, multidisciplinary approach.

$A_{\text {temporary loss of consciousness during a }}^{43 \text { year-old woman experienced a }}$ family trip. She refused medical intervention and about half an hour later she was found unconscious by her child. Rescue maneuvers resulted ineffective and the death was declared as soon as she reached the emergency room. Anamnestic data revealed that the woman underwent a similar lipothymic attack three days before.

At the autopsy all the organs didn't show significative pathological findings except that a voluminous uterine neoformation, with intramural extension, whitish colour on cut surface and fasciculated and nodular aspect (Fig. 2). Moreover at the inspection of the inferior vena cava was exposed a grey, firm thrombotic mass, approximately $30 \mathrm{~cm}$ in length, occupying the whole lumen of the vein (Fig. 1). The mass was focally but tenaciously adherent to the vein wall, but any pedicle or appendage on vascular wall was observed . The thrombus showed a solid aspect, a grey colour and a partially fasciculated surface. The uterine mass and the trhombus were extensively sampled after $24 \mathrm{~h}$ fixation in $10 \%$ buffered formalin and tissue samples were embedded in paraffin blocks. Histological evaluation was performed by two pathologists. Haematoxylin and eosin slides of the uterine mass showed variously twisted bundles of spindle cells without cellular atypia or necrosis (Fig. $3 \mathrm{~A}$ ); a low mitotic rate was reported ( $<1$ mithosis in $50 \mathrm{HPF}$ ). These data favoured a diagnosis of uterine leiomyoma. The thrombotic mass presented the same histological characteristics with extensive hyaline fibrosis associated (Fig. $3 \mathrm{~B}$ and C). Such findings suggested the diagnosis of intravascular leiomyoma. Complementary studies on intravascular mass specimen with immunohistochemical myogenic markers were performed; paraffin sections of

1) "Tor Vergata" University of Rome, Department of Surgery and Experimental Medicine, Rome, Italy

2) "Tor Vergata" University of Rome, Department of Biomedicine and Prevention

3) Sapienza University, Department of Public Health and Infectious Diseases, Rome, Italy

* Corresponding author: MD, Department of Public Health and Infectious Diseases Sapienza University, P.le Aldo Moro 5, 00185 Rome, Italy, Tel/Fax: +3906 4991 4667, Email: giuseppe.messano@uniroma1.it 


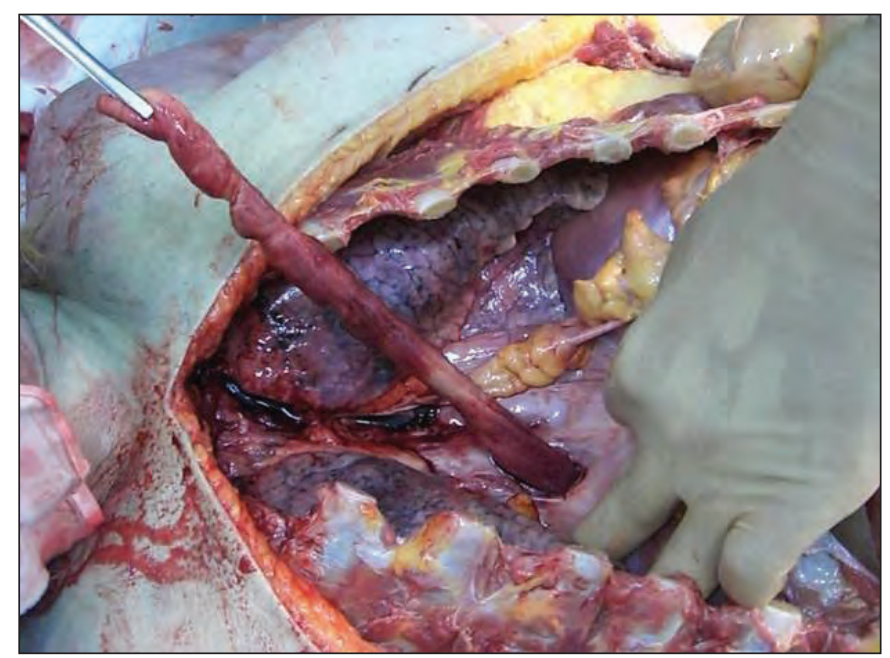

Figure 1. Inferior vena cava: a grey and firm thromotic mass occupies the lumen.

$4-\mu \mathrm{m}$-thick were cut both from diagnostic blocks, and were processed by the Bench Mark automatized system (Ventana, Tucson, AZ, USA).

After pretreatment, sections were incubated with rabbit monoclonal anti-vimentin (clone V9; Ventana, Tucson, AZ, USA; pre-diluted), alpha-smooth muscle actin (clone 1 A 4; Ventana, Tucson, AZ, USA) and desmin (clone DE-R-11, Ventana, Tucson, AZ, USA). Immunohistochemical profile showed positivity for alpha-smooth muscle actin and desmin with negativity for vimetin, confirming the presence of a smooth muscle constituent (Fig. 3 D-G). Furthermore: intracaval mass showed immunohistochemical negativity for HMB-45 (clone HMB-45, Anti-Melanoma; Ventana, Tucson, AZ, USA) (Fig. $3 \mathrm{H}$ ).

\section{DISCUSSION}

Metastasizing intravenous leiomyoma is a rare neoplasm characterized by a benign smooth muscle cell tumor, leiomyoma, proliferating inside pelvic veins or vena cava. Two main theories justify its origin: it may arise from vascular walls within the myometrium or it is the result of a metastatic diffusion from myometrial leiomyoma [1].

This tumor affects particularly middle-aged women (44 years) with a history of uterine leiomyomata or hysterectomy [2].

An intravenous leiomyoma usually reaches the lumen of iliac veins and grows inside inferior vena cava; some cases of lung, lymphnodes and heart chambers' invasion are reported [3]. In latter case, it may result not only in cardiac symptoms or murmur, but even in sudden death [4]. Malignant behaviour is also reported by some authors $[7,8]$, describing intravenous leiomyoma as a low-grade metastasis from leiomyosarcoma. The immunohistochemical profile usually shows positivity for alpha-smooth muscle actin and desmin, but estrogen, vimentin and HMB-45 expression have also been

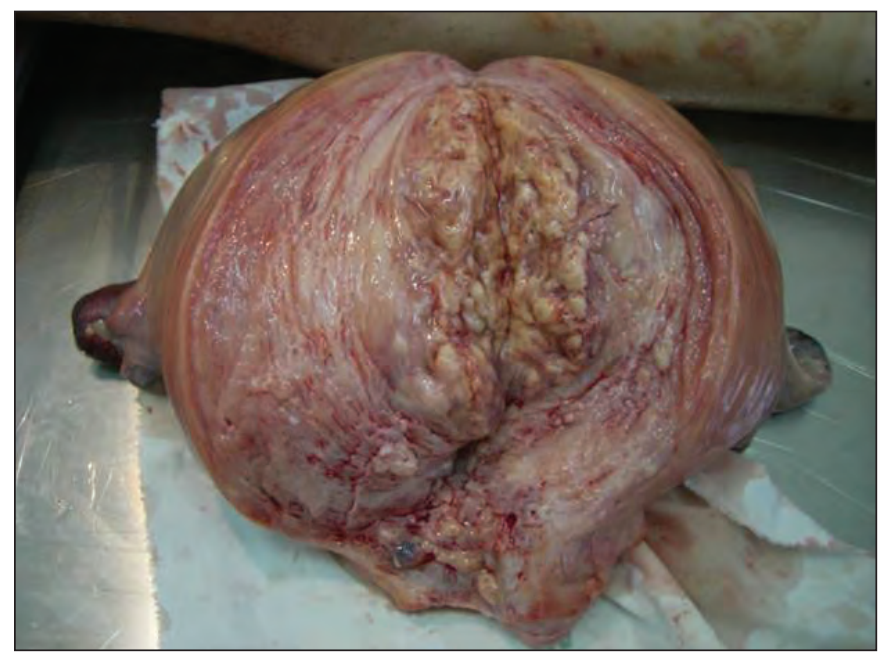

Figure 2. Voluminous uterine neoformation with nodular and fasciculated aspect.

demonstrated [1,9]. In particular: immunoreactivity to HMB-45 in smooth muscle cells belonging to hamartomatous neoplasms has been largely described [5] and some authors describe benign metastasizing leiomyoma as an hamartomatous primitive neoplasm [6] accentuating melanocytic markers positivity. In our experience the neoplasm resulted negative to HMB-45 antibody, ruling out hamartoma from our diagnosis.

In the present case, we described a metastasizing intravenous leiomyoma, likely to originate from a preexistent uterineleiomyoma. Morphological characteristics (contemporary existence of uterine leiomyomatous mass, lack of pedicle or any macroscopically evident point of origin from wall of inferior vena cava) and immunohistochemical phenotype are stackable as a metastazing uterine leiomyoma.

Kwok et al. describe sudden death as: an unexpected natural death within a short time period, generally 1 hour or less from the onset of symptoms; or a non-witnessed death discovered within 24 hours in someone without prior symptoms, or any prior condition that would appear fatal [10]. The main causes of such disease relate to heart: coronary or hypertensive heart diseases are the most common.

For what concerns legal medicine issues, we underline how sometimes it is not possible to state an exact cause of sudden death at autopsy time without resorting to histopathological investigation. In fact at first sight, in our case, autoptic examination suggested a clear post-mortem diagnosis: an inferior vena cava obstruction occurred, consequently causing abrupt cardiac arrest. But only histhological and immunohistochemical examination of the thrombotic mass found in inferior vena cava revealed its leiomyomatous nature. As reported before, an intravenous leiomyoma may cause sudden death: lack of early and pathognomonic symptoms represent a clinicopathologic challenge, causing diagnosis delay of an extensive intravenous invasion until, possibly, sudden death. Considering 

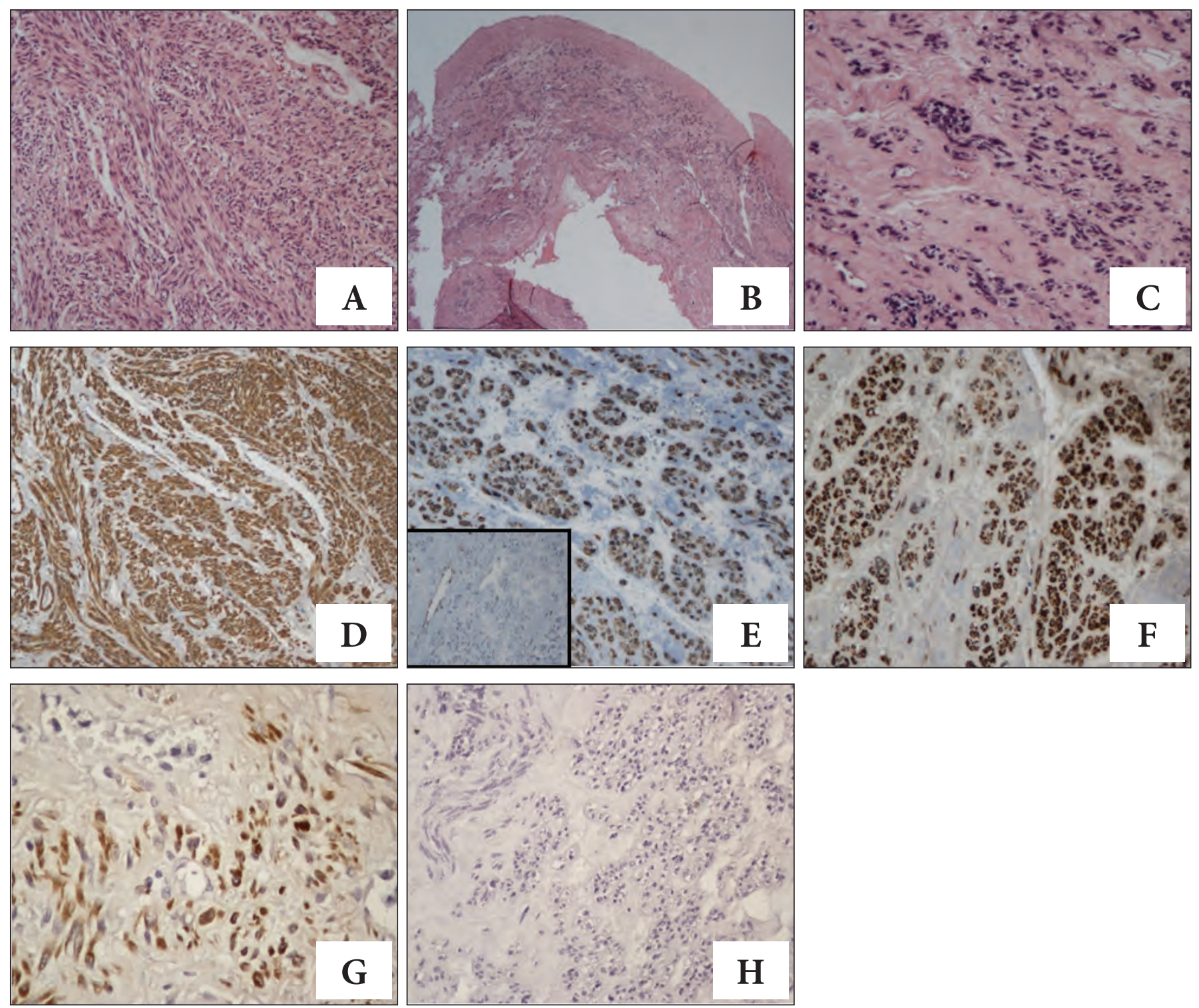

Figure 3. A- uterine leiomyoma with variously twisted bundles of spindle cells, atypia and necrosis are absent. B, C - thrombotic mass in inferior vena cava: extensive hyaline fibrosis associated to twisted bundles of spindle cells. D- immunohistochemical positivity for alpha-smooth muscle actin in uterine leiomyoma. E, F- positivity for alpha-smooth muscle actin and vimentin negativity (inset on E) corroborate the leiomyomatous nature of intracaval thrombotic mass. G- immunohistochemical positivity for desmin in intracaval mass. $\mathrm{H}$ - immunohistochemical negativity for HMB-45 excludes the hamartomatous nature of the intracaval mass.

age and possible risk factors (most of them relate to coronary artery disease) [10] relating to our patient's clinical history, a simple diagnosis of inferior vena cava thrombosis results inadequate to explain her sudden death. In our experience, we can thus join legal medicine investigations, anamnesis collection and histopatologic examinations to realistically suppose that intravenous leiomyomatous mass has asymptomatically grown inside inferior vena cava; fibrin deposit progressively enhanced lumen obstruction until critical symptoms of thrombotic obstruction occurred.
Although intravenous leiomyomatosis is rare, most cases remain unknown and misdiagnosed; a clear diagnosis is usually incidental due to the lack of peculiar features. A sudden cardiac death occurring in middle aged women with history of leiomyomata or hysterectomy should always be investigated and intravenous leiomyoma should be considered as a possible cause. Anatomopathologic report, together with a multidisciplinary approach, remains the main and most useful tool to create a "diagnostic culture" in order to enhance possibilities to prevent sudden cardiac death.

\section{References}

1. Veysel Kutay, Mustafa Tuncer, Mustafa Harman, Hasan Ekim and Cevat Yakut. Intracardiac extension of intravenous leiomyoma. Texas Heart Institute; Vol. 32, N.2, 2005. 
2. Marcia Maria Morales, Alexandre Anacleto, João Carlos Leal, Sérgio Carvalho, Jerônimo Del'ArcoIntravascular leiomyoma with heart extension. Clinics 2012; 67(1): 83-87.

3. Harris LM, Karakousis CP. Intravenous leiomyomatosis with cardiac extension: tumor thrombectomy through an abdominal approach. J Vasc Surg 2000; 31: 1046-1051.

4. Xu ZF, Yong F, Chen YY, Pan AZ. Uterine intravenous leiomyomatosis with cardiac extension: Imaging characteristics and literature review. World J Clin Oncol 2013 February 10; 4(1): 25-28.

5. Maurizio Pea, Franco Bonetti, Giuseppe Zamboni, Guido Martignoni, Maurizio Riva, Romano Colombari, Aldo Mombello, Mariella Bonzanini, Aldo Scarpa, Claudio Ghimenton, and Luciano Fiore Donati. Melanocyte-Marker-HMB-45 is regularly expressed in angiomyolipoma of the kidney. Pathology 1991, 23: 85-188.

6. Po-Chi Huang, Jung-Ta Chen, Chou Chia-Man, Po-Cheung Kwan and William L Ho. Benign metastasizing leiomyoma of the lung: a case report. J Formos Med Assoc. 2000 Dec; 99(12):948-951.

7. Lee HJ, Choi J, Kim KR. Pulmonary benign metastasizing leiomyoma associated with intravenous leiomyomatosis of the uterus: clinical behavior and genomic changes supporting a transportation theory. Int J Gynecol Pathol 2008; 27: 340-345.

8. Lee S, Kim DK, Narm KS, Cho SH. Pulmonary artery embo- lization of intravenous leiomyomatosis extending into the right atrium. Korean J Thorac Cardiovasc Surg 2011; 44: 243-246.

9. Sentinelli S, Covello R, Benevolo M, Licci S, Perrone Donnorso R. Benign metastizing leiomyoma in the lung: report of a case and review of literature. Pathologica (2002) 94: 253-256.

10. Kwok KM, Lee KL, Lau CP, Tse HF. Sudden cardiac death: prevention and treatment. Hong Kong Med J. 2003 Oct;9(5):357-362. 